Upcoming blood and urine analysis showed increased serum creatinine with proteinuria $1 \mathrm{~g} /$ day and hematuria. Following interdisciplinary consultation immunologic investigations were performed showing elevated titres of ANA, anti-dsDNA and decreased complement levels.

The patient was diagnosed with SLE with shrinking lung and active renal involvement and was referred to Rheumatology Unit where treatment was initiated with prednisone $0.5 \mathrm{mg} / \mathrm{kg} /$ day and mycophenolate mofetil $2 \mathrm{~g} /$ day. Respiratory symptoms as well as pulmonary function tests improved within some days after initiation of steroid treatment.

After 12 months the patient displayed still abnormally restrictive respiratory pattern at pulmonary function tests, despite clinical improvement. She was also displaying proteinuria $0.8 \mathrm{~g} /$ day with active urinary sediment and rising anti-dsDNA antibodies. Thus, she underwent Rituximab $1 \mathrm{~g} 2$ weeks apart with subsequent improvement of both renal and respiratory signs and symptoms. Improvement remained stable at 2 years.

Disclosure of Interest: None declared

DOI: 10.1136/annrheumdis-2018-eular.7696

\section{SP0100 NEUROPSYCHIATRIC LUPUS OR NOT?}

A. Fanouriakis. Rheumatology and Clinical Immunology, 4th Department of Internal Medicine, "Attikon" University Hospital, Athens, Greece

A 30 year old woman with a 10 year history of SLE was admitted in the Emergency Department with severe headache, drowsiness and vertigo. Brain magnetic resonance imaging revealed an extensive ischaemic stroke in the cerebellum. During the month prior to the episode, the patient had not been feeling well, complaining for arthralgias, fatigue, hair-loss, low grade-fever and the progressively deteriorating headache.

Should this patient's ischaemic stroke be attributed to lupus? Moreover, if yes, does it represent a thrombotic or an inflammatory process?

The second patient, a 38 year old woman of Asian descent, was referred to our Unit due to refractory seizures and status epilepticus for the past two years. On initial presentation, she had fever, mild leukopenia, low C3 and C4 levels and positive ANA (titer 1:320). She had been previously treated by different neurologists, with a combination of antiepileptic drugs, with no success. Brain magnetic resonance imaging was repeatedly unremarkable and electroencephalogram revealed mild epileptiform changes.

Does this patient represent a case of bona fide neuropsychiatric lupus?

Disclosure of Interest: None declared

DOI: 10.1136/annrheumdis-2018-eular.7726

FRIDAY, 15 JUNE 2018

\section{Understanding the language of basic research, epidemiology and health services articles}

\section{SP0101 UNDERSTANDING THE LANGUAGE OF EPIDEMIOLOGY ARTICLES}

W.G. Dixon. The University of Manchester, Manchester, UK

'Clinical and Epidemiological Research' is one of two headings for original articles in the Annals of Rheumatic Disease. But what is epidemiological research? What do all those different types of bias mean? And what do all of those bizarre statistical terms mean? How can I make sense of the research, and how is it relevant to my practice? Answer: Attend the lecture and find out.

Disclosure of Interest: W. Dixon Consultant for: Google, Bayer

DOI: 10.1136/annrheumdis-2018-eular.7801

\section{\begin{tabular}{|l|l}
\hline SP0102 & UNDERSTANDING THE LANGUAGE OF HEALTH
\end{tabular} SERVICES ARTICLES}

\section{Carmona. IMusc, Madrid, Spain}

The only difference of Health services Research with other types of research is the study unit observed. This goes beyond individuals and it may likely refer to doctors, their clinics, or even countries. We still need to work on hypotheses, although some exploratory research can be done; and we always need to start with a research question. If you are unable to find a research question in an original article, remember: stop reading. We will review some of the top questions answered by health services research, such as performance of doctors and services, efficacy of complex interventions, and inequalities. In addition, we will review basic concepts, such as what is an indicator, very frequently used as outcome measures, or what makes a best practice, as well as some special difficulties, such as multilevel (AKA hierarchical) models or instrumental variables. To end, we will review basic concepts in qualitative research, needed to understand the papers dealing with health services research.

Disclosure of Interest: None declared

DOI: 10.1136/annrheumdis-2018-eular.7739

FRIDAY, 15 JUNE 2018

Joint EULAR - ESSR session on: The role of MR
imaging in rheumatic diseases and its clinical
implications.

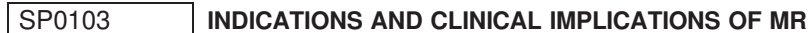 IMAGING IN RA}

B. Ostendorf. Rheumatology, Dept. of Rheumatology and Hiller-Institute for Experimental Rheumatology, Düsseldorf, Germany

In clinical practice, rheumatologists most frequently use imaging techniques to explore bone and soft-tissue involvement in RA. MRI is the best noninvasive observer-independent imaging modality technique that has advantages over clinical examination and conventional radiographs for assessing joint damage and soft tissue inflammation, which are common features even in the earliest stages of RA. MRI provides multiplanar images with a high degree of resolution without ionising radiation. Based on these characteristics, MRI identifies early signs of arthritis where other imaging modalities failed. Main indications for MRI in RA consequently are to determine joint involvement, differential diagnosis and early diagnosis of inflammation, such as synovial changes, changes in tendon sheaths and bursae, as well as bone marrow oedema (BME), not detected by clinical examination, BME even also not by ultrasound. The presence of BME has added benefits to modern diagnostic criteria, and anti-citrullinated peptide antibody positive patients have demonstrated higher osteitis scores. Additionally, MRI helps to assess and define prognosis and outcome, because synovitis and BME are risk factors for the progression of structural changes. Growing data on the validity of $\mathrm{MRI}$ in predicting and assessment of treatment response are available as well. Recent evidence has demonstrated that MRI inflammatory parameters are frequent findings in RA with clinical remission and low disease activity states, which has impact on treatment changes. MRI helps to identify at-risk individuals with arthralgia without clinical arthritis, furthermore these patients with defined RA and high risk for disease progression leading to therapy escalation and also may limit unnecessary treatment with potentially expensive biologic drugs. Studies that directly assess how MRI use in clinical care might even influence decision making, quality of care and cost effective delivery of that care. Clinical studies answering these questions of regular use of MRI are warranted.

Disclosure of Interest: None declared

DOI: 10.1136/annrheumdis-2018-eular.7679

\section{SP0104 INDICATIONS AND CLINICAL IMPLICATIONS OF MAGNETIC RESONANCE IMAGING IN SPONDYLOARTHRITS}

M. Østergaard. Copenhagen Center for Arthritis Research, Copenhagen University Hospital Rigshospitalet, Glostrup, Denmark

Axial spondyloarthritis (axSpA) is a chronic inflammatory disease that affects the sacroiliac joints and the spine. Whereas early disease is characterised by inflammation, severe structural damage may occur later in the disease course.

Magnetic resonance imaging (MRI) can detect inflammatory lesions (bone marrow oedema (BME)/osteitis), and structural lesions (erosions, bone spurs, ankylosis and fat metaplasia).

In patients suspected for axial spondyloarthritis (axSpA) in clinical practice, MRI has a key role for early diagnosis of axial $\mathrm{SpA}$, since MRI is by far the best available method for early detection of inflammation in the spine and sacroiliac joints $\mathrm{MRI}$ is also more sensitive and accurate for assessment of structural sacroiliac changes than conventional radiography. Differential diagnoses, including anatomical variations, degenerative disease, osteitis condensans ilii, infections and others, should of course always be considered.

In patients with diagnosed axial $\mathrm{SpA}, \mathrm{MRI}$ is the method of choice for sensitive objective monitoring of axial inflammation, and also peripheral inflammation and damage can be assessed. Whole-body MRI is a promising method, which may become important for simultaneous assessment of axial and peripheral disease manifestations in one examination. Furthermore, MRI can provide information which may help predict the response to therapy and the risk of subsequent progression of structural damage. 